Research Article

\title{
Best Proximity Point Theorems for Cyclic Contractions Mappings in Banach Algebras
}

\author{
K. Chaira, ${ }^{1}$ S. Chaira, ${ }^{2}$ and S. Lazaiz $(\mathbb{D})^{3}$ \\ ${ }^{1}$ L3A Laboratory, Department of Mathematics and Computer Sciences, Faculty of Sciences Ben M'sik, \\ University of Hassan II Casablanca, Morocco \\ ${ }^{2}$ Laboratory of Mathematics and Applications, Faculty of Sciences and Technologies, Mohammedia, \\ University Hassan II Casablanca, Morocco \\ ${ }^{3}$ LASMA Laboratory, Department of Mathematics, Faculty of Sciences Dhar El Mahraz, University Sidi Mohamed Ben Abdellah, \\ Fes, Morocco
}

Correspondence should be addressed to S. Lazaiz; samih.lazaiz@usmba.ac.ma

Received 26 August 2020; Revised 12 September 2020; Accepted 27 September 2020; Published 5 November 2020

Academic Editor: Huseyin Isik

Copyright $\odot 2020 \mathrm{~K}$. Chaira et al. This is an open access article distributed under the Creative Commons Attribution License, which permits unrestricted use, distribution, and reproduction in any medium, provided the original work is properly cited.

In this paper, we present some new best proximity point theorems for three operators acting in Banach algebras. An application is given to show the usefulness and the applicability of the obtained results.

\section{Introduction}

The study of functional integral equations and differential equations is the main object of research in nonlinear functional analysis. These equations occur in physical, biological, and economic problems. Some of these equations can be formulated into nonlinear operator equations:

$$
x=A(x) \cdot B(x)+C(x)
$$

in suitable Banach algebras.

Recently, many authors are interested on the study of equation (NOE) and obtained some interesting results (see for instance [1-5]). In 2010, Ben Amar et al. [6] proved some existence fixed point theorems which allowed them to solve equation (NOE) where the involved operators are weakly sequentially continuous.

Let $X$ be a Banach algebra with a norm $\|\cdot\|$. Let $\left(\Omega_{1}, \Omega_{2}\right)$ be a pair of nonempty subsets of $X$. Given two mappings $A$ and $C$ defined on $X$ and an operator $B: \Omega_{1} \cup \Omega_{2} \longrightarrow X$. Under suitable conditions, we define the operator $T:=$ $((I-C) / A)^{-1} \circ B: \Omega_{1} \cup \Omega_{2} \longrightarrow \Omega_{1} \cup \Omega_{2}$ such that $T\left(\Omega_{1}\right) \subset$ $\Omega_{2}$ and $T\left(\Omega_{2}\right) \subset \Omega_{1}$. If $\Omega_{1} \cap \Omega_{2}$ is nonempty, then the mapping $T$ restricted to $\Omega_{1} \cap \Omega_{2}$ is a self mapping. Then, a solu- tion of equation (NOE) is a fixed point of $T$. Furthermore, if the fixed point equation $x=A(x) \cdot B(x)+C(x)$ does not possess a solution it is natural to explore to find an $x^{*} \in \Omega_{1}$ satisfying

$$
\left\|x^{*}-T\left(x^{*}\right)\right\|=\operatorname{dist}\left(\Omega_{1}, \Omega_{2}\right)
$$

where $\operatorname{dist}\left(\Omega_{1}, \Omega_{2}\right)=\inf \left\{\|x-y\|: x \in \Omega_{1}, y \in \Omega_{2}\right\}$. This point $x^{*} \in \Omega_{1}$ is said to be the best proximity point of $T$. Note that a point $x \in \Omega_{1} \cup \Omega_{2}$ is the best proximity point of $T$ if $x$ is a solution of the minimization problem

$$
\min _{x \in \Omega_{1} \cup \Omega_{2}} d(x, T x) .
$$

The best proximity point notion can be viewed as a generalization of fixed point, since most fixed point theorems can be derived as corollaries of the best proximity point theorems.

The first result of this kind is due to Fan (see [7], Theorem 2) which is stated in normed spaces for continuous mappings. In [8], Eldred and Veeramani introduced the concept of cyclic contraction mappings and gave the best proximity point results for this class of mappings. They also gave an algorithm to reach this best proximity point where the space 
is uniformly convex. Furthermore, in [9], Taghafi and Shahzad proved the existence of the best proximity point for a cyclic contraction mapping in a reflexive Banach space.

For noncyclic mappings, i.e., $T\left(\Omega_{1}\right) \subset \Omega_{1}$ and $T\left(\Omega_{2}\right) \subset$ $\Omega_{2}$, Gabelah and Künzi in [10] established some best proximity point results in the framework of complete CAT(0) spaces. In addition, they gave an approach to reach this best proximity point by means of an algorithm. Regarding the relationship between the noncyclic and cyclic results, the authors in [11] proved that the existence of best proximity points for cyclic nonexpansive mappings is equivalent to the existence of best proximity pairs for noncyclic nonexpansive mappings in the setting of strictly convex Banach spaces. For more on the best proximity point results, the interested reader can consult [12-18].

The paper is organized as follows. After some preliminaries, in Section 3, we prove the existence of the best proximity point where the involving operators are $\mathscr{D}$-Lipschitzs and cyclic contraction (see Theorem 1). Also, an example is given to illustrate the obtained result. In Theorem 1, we consider the case where $X$ is a uniformly convex Banach algebra. In Section 4, we show the applicability of our result (Theorem 1) to the theory of nonlinear integral equations:

$$
\begin{aligned}
& x(t)=K(t, x(t))+(T x)(t) \cdot\left(q_{2}+\int_{0}^{t} g(s, y(s)) d s\right) \\
& y(t)=K(t, y(t))+(T y)(t) \cdot\left(q_{1}+\int_{0}^{t} f(s, x(s)) d s\right),
\end{aligned}
$$

where $\Omega_{1}$ and $\Omega_{2}$ two subsets of the Banach algebra $E=$ $\mathscr{C}(J, X)$ of all continuous functions from $J$ to $X$.

\section{Preliminaries}

Definition 1. An algebra $X$ is a vector space endowed with an internal composition law noted by (.) i.e.,

$$
\left\{\begin{array}{l}
(\cdot): X \times X \longrightarrow X \\
(x, y) \longrightarrow x \cdot y
\end{array}\right.
$$

which is associative and bilinear.

A normed algebra is an algebra endowed with a norm satisfying the following property for all $x, y \in X ;\|x \cdot y\| \leq\|x\| \|$ $y \|$. A complete normed algebra is called a Banach algebra.

Definition 2. Let $X$ be a Banach space with norm $\|\cdot\|$. A mapping $T: X \longrightarrow X$ is called $\mathscr{D}$-Lipschitz if there exists a continuous nondecreasing function $\Phi_{T}: \mathbb{R}_{+} \longrightarrow \mathbb{R}_{+}$satisfying

$$
\|T x-T y\| \leq \Phi_{T}(x-y)
$$

for all $x, y \in X$ with $\Phi_{T}(0)=0$. In the special case when $\Phi_{T}(r)=\alpha r$ for some $\alpha>0, T$ is called lipschitzian mapping with a Lipschitz constant $\alpha$.
Definition 3. Let $(X,\|\cdot\|)$ be a Banach space. We say that $X$ is uniformly convex if for every $\varepsilon>0$,

$\delta(\varepsilon)=\inf \left\{1-\left\|\frac{x+y}{2}\right\|:\|x\| \leq 1,\|y\| \leq 1\right.$ and $\left.\|x-y\| \geq \varepsilon\right\}>0$,

The function $\delta$ is known as the modulus of uniform convexity of $X$. Note that any uniformly convex Banach space is reflexive.

Theorem 4 (see [17]). Let $X$ be a uniformly convex Banach space. Let $\Omega_{1}$ be a nonempty closed bounded convex subset of $X$ such that $\Omega_{1}^{0}$ is compact, and $\Omega_{2}$ be a nonempty closed convex subset of $X$. Let $T: \Omega_{1} \cup \Omega_{2} \longrightarrow \Omega_{1} \cup \Omega_{2}$ be a relatively nonexpansive mapping. Then, there exists $x^{*} \in \Omega_{1}$ such that $\left\|x^{*}-T\left(x^{*}\right)\right\|=\operatorname{dist}\left(\Omega_{1}, \Omega_{2}\right)$.

The authors in [8] introduced the following notion of cyclic contraction.

Definition 5. Let $\Omega_{1}$ and $\Omega_{2}$ be nonempty subsets of a metric space $X$. A mapping $T: \Omega_{1} \cup \Omega_{2} \longrightarrow \Omega_{1} \cup \Omega_{2}$ is said to be a cyclic contraction if it satisfies:

(1) $T\left(\Omega_{1}\right) \subset \Omega_{2}$ and $T\left(\Omega_{2}\right) \subset \Omega_{1}$

(2) for some $k \in(0,1), d(T(x), T(y)) \leq k d(x, y)+(1-$ k) $\operatorname{dist}\left(\Omega_{1}, \Omega_{2}\right)$, for all $x \in \Omega_{1}, y \in \Omega_{2}$

Since $\operatorname{dist}\left(\Omega_{1}, \Omega_{2}\right) \leq d(x, y)$, for $x \in \Omega_{1}$ and $y \in \Omega_{1}, d(T$ $(x), T(y)) \leq d(x, y)$ for all $x \in \Omega_{1} y \in \Omega_{2}$, i.e., $T$ is relatively nonexpansive.

We conclude this section by recalling some best proximity point results for this class of mappings.

Theorem 6 (see [8]). Let $\Omega_{1}$ and $\Omega_{2}$ be nonempty closed subsets of a complete metric space $X$. Let $T: \Omega_{1} \cup \Omega_{2} \longrightarrow$ $\Omega_{1} \cup \Omega_{2}$ be a cyclic contraction mapping and $x_{0} \in \Omega_{1}$. Define $x_{n+1}=T\left(x_{n}\right), n \in \mathbb{N}$. Suppose $\left\{x_{2 n}: n \in \mathbb{N}\right\}$ has a convergent subsequence in $\Omega_{1}$, then there exists $x \in \Omega_{1}$ such that $d(x$, $T(x))=\operatorname{dist}\left(\Omega_{1}, \Omega_{2}\right)$.

Theorem 7 (see $[8,19]$ ). Let $\Omega_{1}$ and $\Omega_{2}$ be nonempty closed convex subsets of a uniformly convex Banach space. Suppose $T: \Omega_{1} \cup \Omega_{2} \longrightarrow \Omega_{1} \cup \Omega_{2}$ is a cyclic contraction mapping. Then, $T$ has a unique best proximity point in $\Omega_{1}$. Further, if $x_{0} \in \Omega_{1}$ and $x_{n+1}=T\left(x_{n}\right)$, then the sequence $\left(x_{2 n}\right)_{n \geq 0}$ converges to the best proximity point.

\section{Main Results}

We start this section by introducing the notion of $(\alpha$, $\beta)$-monotone property for a pair of functions.

Definition 8. Let $(\alpha, \beta) \in\left(\mathbb{R}_{+}\right)^{2}$ and $\phi, \psi: \mathbb{R}_{+} \longrightarrow \mathbb{R}_{+}$be two mappings. We say that the pair $(\phi, \psi)$ has the property $(\alpha$, $\beta)$-monotone if 
(i) $\phi(0)=0=\psi(0)$,

(ii) $I-\alpha \cdot \phi-\beta \cdot \psi$ is nondecreasing on $\mathbb{R}_{+}$and $\lim _{r \rightarrow+\infty}(r-\alpha \phi(r)-\beta \psi(r))=+\infty$

Remark 9. If $(\phi, \psi)$ has the property $(\alpha, \beta)$-monotone and $\phi, \psi$ are continuous, then the mapping $I-\alpha \cdot \phi-\beta \cdot \psi: \mathbb{R}_{+}$ $\longrightarrow \mathbb{R}_{+}$is invertible.

Example 1 . Let $\phi, \psi: \mathbb{R}_{+} \longrightarrow \mathbb{R}_{+}$be the mappings defined by:

$$
\phi(r)=\frac{r}{r+1} \text { and } \psi(r)=\frac{r}{2}, \text { for all } r \in \mathbb{R}_{+} .
$$

So, the mapping $I-1 / 2 \phi-\psi: \mathbb{R}_{+} \longrightarrow \mathbb{R}_{+}$has the property $(1 / 2,1)$-monotone.

Recall that an operator $A$ from a Banach algebra $X$ is said to be regular on $X$ if $A$ maps $X$ into the set of all invertible elements of $X$.

Theorem 10. Let $\left(\Omega_{1}, \Omega_{2}\right)$ be a nonempty closed pair of a Banach algebra $X$. Let $A, C: X \longrightarrow X$ and $B: \Omega_{1} \cup \Omega_{2} \longrightarrow$ $X$ be three operators which satisfy the following conditions:

(1) $A$ is regular on $X$ and $\|A\|<1$

(2) $A$ and $C$ are $\mathscr{D}$-Lipschitzs with the $\mathscr{D}$-functions $\Phi_{A}$ and $\Phi_{C}$, respectively, $B\left(\Omega_{1} \cup \Omega_{2}\right)$ is bounded with bound $M$, and $M \Phi_{A}(r)+\Phi_{C}(r) \leq(1-\|A\|) r$ for all $r>0$, and $I-M \Phi_{A}-\Phi_{C}$ is nondecreasing

(3) $B$ is cyclic contraction mapping on $\Omega_{1} \cup \Omega_{2}$

(4) suppose there exists a sequence $\left(x_{n}\right)_{n \geq 0}$ of $\Omega_{1} \cup \Omega_{2}$ such that $x_{0} \in \Omega_{1}, B\left(x_{n}\right)=((I-C) / A)\left(x_{n+1}\right)$ and the sequence $\left(x_{2 n}\right)_{n \geq 0}$ has a convergent subsequence in $\Omega_{1}$,

(5)

$\left(\begin{array}{l}y=A(y) \cdot B(x)+C(y), x \in \Omega_{1} \Rightarrow y \in \Omega_{2} \\ x=A(x) \cdot B(y)+C(x), y \in \Omega_{2} \Rightarrow x \in \Omega_{1}\end{array}\right.$

Then, there exists $(x, y) \in \Omega_{1} \times \Omega_{2}$ such that

$$
\begin{aligned}
& \left\|\frac{x-A(x) \cdot B(x)-C(x)}{A(x)}\right\| \\
& \quad=\operatorname{dist}\left(\Omega_{1}, \Omega_{2}\right)=\left\|\frac{y-A(y) \cdot B(y)-C(y)}{A(y)}\right\| .
\end{aligned}
$$

Proof. Let $y$ be fixed in $\Omega_{1} \cup \Omega_{2}$ and let us define the mapping $F_{y}$ on $X$ by

$$
F_{y}(x)=A(x) \cdot B(y)+C(x), \text { for all } x \in X
$$

Let $x_{1}, x_{2} \in X$. The use of assumption (ii) leads to

$$
\begin{aligned}
\left\|F_{y}\left(x_{1}\right)-F_{y}\left(x_{2}\right)\right\| \leq & \left\|A\left(x_{1}\right) \cdot B(y)-A\left(x_{2}\right) \cdot B(y)\right\| \\
& +\left\|C\left(x_{1}\right)-C\left(x_{2}\right)\right\| \\
\leq & \left\|A\left(x_{1}\right)-A\left(x_{2}\right)\right\|\|B(y)\| \\
& +\left\|C\left(x_{1}\right)-C\left(x_{2}\right)\right\| \\
\leq & M \Phi_{A}\left(\left\|x_{1}-x_{2}\right\|\right) \\
& +\Phi_{C}\left(\left\|x_{1}-x_{2}\right\|\right)
\end{aligned}
$$

Now an application of Boyd and Wong's fixed point theorem [20], Theorem 1 leads to the existence of a unique point $x_{y} \in X$ such that $F_{y}\left(x_{y}\right)=x_{y}$. Hence, the operator $T:=$ $((I-C) / A)^{-1} B: \Omega_{1} \cup \Omega_{2} \longrightarrow X$ is well defined.

Moreover, assumption (v) implies that $T\left(\Omega_{1}\right) \subset \Omega_{2}$ and $T\left(\Omega_{2}\right) \subset \Omega_{1}$. Indeed, let $x \in \Omega_{1}$ and $y \in X$ such that $y=A(y)$ $. B(x)+C(y)$, so $T(x)=((I-C) / A)^{-1} B(x)=y \in \Omega_{2}$. Similarly, for all $y \in \Omega_{2}, T(y) \in \Omega_{1}$. Hence, $T$ is cyclic on $\Omega_{1} \cup$ $\Omega_{2}$.

$T$ is cyclic contraction on $\Omega_{1} \cup \Omega_{2}$. Indeed, let $(x, y)$ $\in \Omega_{1} \times \Omega_{2}$, the use of assumption (ii) and (iii) and the fact that $T(z)=A(T(z)) \cdot B(z)+C(T(z))$ for all $z \in \Omega_{1} \cup$ $\Omega_{2}$ leads to

$$
\begin{aligned}
\|T(x)-T(y)\| \leq & \|A(T(x)) \cdot B(x)-A(T(y)) \cdot B(y)\| \\
& +\| C(T(x)-C(T(y) \| \\
\leq & \|A(T(x))-A(T(y))\|\|B\| \\
& +\|B(x)-B(y)\|\|A\| \\
& +\| C(T(x)-C(T(y) \| \\
\leq & \|A\|\left(k\|x-y\|+(1-k) \operatorname{dist}\left(\Omega_{1}, \Omega_{2}\right)\right) \\
& +M_{A}(\|T(x)-T(y)\|) \\
& +\Phi_{C}(\|T(x)-T(y)\|
\end{aligned}
$$

Since $\left(\Phi_{A}, \Phi_{C}\right)$ has the property $(M, 1)$-monotone, we have

$$
\begin{aligned}
\|T(x)-T(y)\| \leq & \left(I-M \Phi_{A}-\Phi_{C}\right)^{-1} \\
& \cdot(\|A\|(k\|x-y\|+(1-k) \operatorname{dist}(A, B))) \\
\leq & k\|x-y\|+(1-k) \operatorname{dist}\left(\Omega_{1}, \Omega_{2}\right) .
\end{aligned}
$$

By (iv), there exists a sequence $\left(x_{n}\right)_{n>0}$ of $\Omega_{1} \cup \Omega_{2}$ such that $x_{0} \in \Omega_{1}, T\left(x_{n}\right)=x_{n+1}$ and the sequence $\left(x_{2 n}\right)_{n \geq 0}$ has a convergent subsequence in $\Omega_{1}$.

Thus, by Theorem 6 , there exists $\left(x_{1}, y_{1}\right) \in \Omega_{1} \times \Omega_{2}$ such that $\left\|x_{1}-T x_{1}\right\|=\operatorname{dist}\left(\Omega_{1}, \Omega_{2}\right)=\left\|y_{1}-T y_{1}\right\|$.

Let $y=((I-C) / A)^{-1}\left(B\left(x_{1}\right)\right) \in \Omega_{2}$. By (iii), $B$ is cyclic contraction on $\Omega_{1} \cup \Omega_{2}, B(y) \in \Omega_{1}$ and $((I-C) / A)(y) \in \Omega_{2}$, so 


$$
\begin{aligned}
\operatorname{dist}\left(\Omega_{1}, \Omega_{2}\right) & \leq\left\|\frac{y-A(y) \cdot B(y)-C(y)}{A(y)}\right\| \\
& =\left\|\left(\frac{I-C}{A}\right)(y)-B(y)\right\| \leq\left\|B\left(x_{1}\right)-B(y)\right\| \\
& \leq\left\|x_{1}-y\right\|=\left\|x_{1}-\left(\frac{I-C}{A}\right)^{-1}\left(B\left(x_{1}\right)\right)\right\| \\
& =\operatorname{dist}\left(\Omega_{1}, \Omega_{2}\right) .
\end{aligned}
$$

Similarly, $\|(x-A(x) \cdot B(x)-C(x)) / A(x)\|=\operatorname{dist}\left(\Omega_{1}, \Omega_{2}\right.$ ) , where $x=((I-C) / A)^{-1}\left(B\left(y_{1}\right)\right) \in \Omega_{1}$.

Example 2. Let $X=\mathbb{R}$ endowed with the usual norm $|\cdot|$ and let $\Omega_{1}=[1 / 6,1 / 4], \Omega_{2}=[3 / 8,1]$.

(i) Let $B$ the function defined on $\Omega_{1} \cup \Omega_{2}$ by

$$
B x=\left(\begin{array}{l}
\frac{3}{8} \text { if } x \in \Omega_{1} \\
\frac{1}{4} \text { if } x \in \Omega_{2} .
\end{array}\right.
$$
$1 / 8$

Let $(x, y) \in \Omega_{1} \times \Omega_{2}$, we have $|x-y|=y-x \geq 3 / 8-1 / 4=$

$$
\begin{aligned}
|B x-B y| & =\left|\frac{3}{8}-\frac{1}{4}\right|=\frac{1}{8}=\operatorname{dist}\left(\Omega_{1}, \Omega_{2}\right) \\
& \leq k \operatorname{dist}\left(\Omega_{1}, \Omega_{2}\right)+(1-k) \operatorname{dist}\left(\Omega_{1}, \Omega_{2}\right) \\
& \leq k|x-y|+(1-k) \operatorname{dist}\left(\Omega_{1}, \Omega_{2}\right), \text { where } k \in[0,1[.
\end{aligned}
$$

Thus, $B$ is cyclic contraction on $\Omega_{1} \cup \Omega_{2}$ and $M=\mid B\left(\Omega_{1}\right.$ $\left.\cup \Omega_{2}\right) \mid=3 / 8$.

(ii) Let $A$ the function defined on $\mathbb{R}$ by $A x=1 / 3$, for all $x \in \mathbb{R}$. The function $A$ is $\mathscr{D}$-Lipschitz with the $\mathscr{D}$ function $\Phi_{A}=0$, and $\|A\|=1 / 3$

(iii) Let $C$ the function defined on $X$ by

$$
C x=\left(\begin{array}{ll}
\frac{1}{6} & \text { if } x \in X \backslash \Omega_{2} \\
\frac{1}{4} & \text { if } x \in \Omega_{2} .
\end{array}\right.
$$

For each $(x, y) \in \Omega_{1} \times \Omega_{2}$,

$$
|C x-C y|=\left|\frac{1}{6}-\frac{1}{4}\right|=\frac{1}{12}=\frac{2}{3} \cdot \frac{1}{8} \leq \frac{2}{3} \cdot|x-y| .
$$

The function $C$ is $\mathscr{D}$-Lipschitz with the $\mathscr{D}$-function defined by $\Phi_{C}(r)=2 / 3 . r$, for all $r \in \mathbb{R}_{+}$. We have $I-M \Phi_{A}$ $-\Phi_{C}=I-\Phi_{C}: r \mapsto 2 / 3 r$ is nondecreasing, and for all $r>0$,

$$
\left(M \Phi_{A}+\Phi_{C}\right)(r)=\frac{2}{3} r \leq(1-\|A\|) r .
$$

(iv) Let $x \in \mathbb{R}$ and $y \in \Omega_{2}$. Suppose $x=A x \cdot B y+C x$ and $y=A y \cdot B x+C y$. We have

$$
\begin{aligned}
& x=A x \cdot B y+C x=\left(\frac{1}{3}\right) \cdot\left(\frac{1}{4}\right)+\frac{1}{6}=\frac{1}{4} \in \Omega_{1}, \\
& y=A y \cdot B x+C y=\left(\frac{1}{3}\right) \cdot\left(\frac{3}{8}\right)+\frac{1}{4}=\frac{3}{8} \in \Omega_{2} .
\end{aligned}
$$

(v) For all $y \in \Omega_{2},((I-C) / A)^{-1}(y)=(y / 3)+1 / 4$, so for each $x \in \Omega_{1},((I-C) / A)^{-1}(B x)=((I-C) / A)^{-1}(3 / 8$ )$=3 / 8$. Thus, for any sequence $\left(x_{n}\right)_{n \geq 0}$ of $\Omega_{1} \cup$ $\Omega_{2}$ such that $x_{0} \in \Omega_{1}, B\left(x_{n}\right)=((I-C) / A)\left(x_{n+1}\right)$, the sequence $\left(x_{2 n}\right)_{n \geq 0}$ has a convergent subsequence

Hence, by Theorem 1 , there exists $(x, y) \in \Omega_{1} \times \Omega_{2}$ such that

$$
\begin{aligned}
& \left\|\frac{x-A(x) \cdot B(x)-C(x)}{A(x)}\right\| \\
& \quad=\operatorname{dist}\left(\Omega_{1}, \Omega_{2}\right)=\left\|\frac{y-A(y) \cdot B(y)-C(y)}{A(y)}\right\| .
\end{aligned}
$$

where $(x, y)=(1 / 4,3 / 8)$.

Theorem 11. Let $\left(\Omega_{1}, \Omega_{2}\right)$ be a nonempty closed pair of a Banach algebra $X$. Let $B: \Omega_{1} \cup \Omega_{2} \longrightarrow X$ and $C: X \longrightarrow X$ be two operators which satisfy the following conditions:

(1) $C$ is $\mathscr{D}$-Lipschitz with the $\mathscr{D}$-function $\Phi_{C}, \Phi_{C}(r)<r$, for all $r>0$

(2) B is cyclic contraction mapping on $\Omega_{1} \cup \Omega_{2}$

(3) $(I-C)^{-1}$ is relatively nonexpansive mapping on $\Omega_{1}$ $\cup \Omega_{2}$

(4) suppose there exists a sequence $\left(x_{n}\right)_{n \geq 0}$ of $\Omega_{1} \cup \Omega_{2}$ such that $x_{0} \in \Omega_{1}, B\left(x_{n}\right)=(I-C)\left(x_{n+1}\right)$ and the sequence $\left(x_{2 n}\right)_{n \geq 0}$ has a convergent subsequence in $\Omega_{1}$

(5) $\left(\begin{array}{l}y=B(x)+C(y), x \in \Omega_{1} \Rightarrow y \in \Omega_{2} \\ x=B(y)+C(x), y \in \Omega_{2} \Rightarrow x \in \Omega_{1}\end{array}\right.$

Then, there exists $(x, y) \in \Omega_{1} \times \Omega_{2}$ such that

$$
\|x-B(x)-C(x)\|=\operatorname{dist}\left(\Omega_{1}, \Omega_{2}\right)=\|y-B(y)-C(y)\| .
$$


Proof. By (i), we show that $T:=(I-C)^{-1} \cdot B: \Omega_{1} \cup \Omega_{2}$ $\longrightarrow X$ is well defined. Moreover, the use of assumptions (ii), (iii), and (v) shows that $T$ is cyclic contraction on $\Omega_{1} \cup \Omega_{2}$. Indeed, by (v), it is cyclic. Let $(x, y) \in \Omega_{1} \times \Omega_{2}$, the use of assumptions (ii) and (iii) leads to $(B(x), B(y))$ $\in \Omega_{2} \times \Omega_{1}$,

$$
\begin{aligned}
\|T(x)-T(y)\|= & \left\|(I-C)^{-1} B(x)-(I-C)^{-1} B(y)\right\| \\
\leq & \|B(x)-B(y)\| \leq k\|x-y\| \\
& +(1-k) \operatorname{dist}\left(\Omega_{1}, \Omega_{2}\right) .
\end{aligned}
$$

By (iv), there exists a sequence $\left(x_{n}\right)_{n \geq 0}$ of $\Omega_{1} \cup \Omega_{2}$ such that $x_{0} \in \Omega_{1}, T\left(x_{n}\right)=x_{n+1}$ and the sequence $\left(x_{2 n}\right)_{n \geq 0}$ has a convergent subsequence in $\Omega_{1}$. that

Thus, by Theorem 6 , there exists $\left(x_{1}, y_{1}\right) \in \Omega_{1} \times \Omega_{2}$ such

$$
\left\|x_{1}-T x_{1}\right\|=\operatorname{dist}\left(\Omega_{1}, \Omega_{2}\right)=\left\|y_{1}-T y_{1}\right\| .
$$

Let $y=(I-C)^{-1}\left(B\left(x_{1}\right)\right) \in \Omega_{2}$. We have $B(y) \in \Omega_{1}$ and $(I-C)(y) \in \Omega_{2}$. By (iii), $B$ is cyclic contraction on $\Omega_{1} \cup \Omega_{2}$, so

$$
\begin{aligned}
\operatorname{dist}\left(\Omega_{1}, \Omega_{2}\right) \leq & \|y-B(y)-C(y)\|=\|(I-C)(y)-B(y)) \| \\
\leq & \left.\| B\left(x_{1}\right)-B(y)\right)\|\leq k\| x_{1}-y \| \\
& +(1-k) \operatorname{dist}\left(\Omega_{1}, \Omega_{2}\right) \leq\left\|x_{1}-y\right\| \\
= & \left\|x_{1}-(I-C)^{-1}\left(B\left(x_{1}\right)\right)\right\|=\operatorname{dist}\left(\Omega_{1}, \Omega_{2}\right)
\end{aligned}
$$

Similarly, we get $\|x-A(x) \cdot B(x)-C(x)\|=\operatorname{dist}\left(\Omega_{1}, \Omega_{2}\right)$, where $x=(I-C)^{-1}\left(B\left(y_{1}\right)\right) \in \Omega_{1}$.

Remark 12. Under the same hypotheses of the previous theorem where $\mathrm{C}=0$, we obtain the classical result. That is, there exists $(x, y) \in \Omega_{1} \times \Omega_{2}$ such that

$$
\|x-B(x)\|=\operatorname{dist}\left(\Omega_{1}, \Omega_{2}\right)=\|y-B(y)\| .
$$

Theorem 13. Let $\left(\Omega_{1}, \Omega_{2}\right)$ be a nonempty closed convex pair of a uniformly convex Banach algebra $X$. Let $A, C: X \longrightarrow X$ and $B: \Omega_{1} \cup \Omega_{2} \longrightarrow X$ be three operators which satisfy the following conditions:

(1) $A$ is regular on $X$ and $\|A\|<1$

(2) A and $C$ are $\mathscr{D}$-Lipschitzs with the $D$-functions $\Phi_{A}$ and $\Phi_{C}$, respectively, $B\left(\Omega_{1} \cup \Omega_{2}\right)$ is bounded with bound $M$, and $M \Phi_{A}(r)+\Phi_{C}(r) \leq(1-\|A\|) r$ for all $r>0$ and $I-M \Phi_{A}-\Phi_{C}$ is nondecreasing

(3) B is cyclic contraction on $\Omega_{1} \cup \Omega_{2}$
(4) $\left(\begin{array}{l}y=A(y) \cdot B(x)+C(y), x \in \Omega_{1} \Rightarrow y \in \Omega_{2} \\ x=A(x) \cdot B(y)+C(x), y \in \Omega_{2} \Rightarrow x \in \Omega_{1} .\end{array}\right.$

Then, there exists a unique $(x, y) \in \Omega_{1} \times \Omega_{2}$ such that

$$
\begin{aligned}
& \left\|\frac{x-A(x) \cdot B(x)-C(x)}{A(x)}\right\| \\
& \quad=\operatorname{dist}\left(\Omega_{1}, \Omega_{2}\right)=\left\|\frac{y-A(y) \cdot B(y)-C(y)}{A(y)}\right\| .
\end{aligned}
$$

Further, if $x_{0} \in \Omega_{1}$ and $B\left(x_{n}\right)=((I-C) / A)\left(x_{n+1}\right), n \in \mathbb{N}$, then the sequence $\left(x_{2 n}\right)_{n \geq 0}$ converges to the best proximity point.

Proof. By (i), (ii), (iii), and (v), we show that $T:=$ $((I-C) / A)^{-1} \cdot B: \Omega_{1} \cup \Omega_{2} \longrightarrow X$ is well defined and cyclic contraction on $\Omega_{1} \cup \Omega_{2}$.

Thus, by Theorem 7 and (iv), there exists a unique $(x, y)$ $\in A \times B$ such that

$$
\begin{aligned}
& \left\|\frac{x-A(x) \cdot B(x)-C(x)}{A(x)}\right\| \\
& \quad=\operatorname{dist}\left(\Omega_{1}, \Omega_{2}\right)=\left\|\frac{y-A(y) \cdot B(y)-C(y)}{A(y)}\right\|,
\end{aligned}
$$

and if $x_{0} \in \Omega_{1}$ with $B\left(x_{n}\right)=((I-C) / A)\left(x_{n+1}\right)$, i.e., $T\left(x_{n}\right)=$ $x_{n+1}$ for all $n \in \mathbb{N}$, then the sequence $\left(x_{2 n}\right)_{n \geq 0}$ converges to the best proximity point.

\section{Application}

Let $E=\mathscr{C}(J, \mathbb{R})$ the Banach algebra of all continuous functions from $J=[0,1]$ to $\mathbb{R}$, endowed with the sup-norm $\|\cdot\|_{\infty}$, defined by

$$
\|x\|_{\infty}=\sup \{|x(t)|, t \in[0,1]\},
$$

for each $x \in C(J, \mathbb{R})$. Let $\left(q_{1}, q_{2}\right) \in \mathbb{R}^{2}$ and suppose $q_{1}<0<q_{2}$. We consider the closed and nonempty sets

$$
\begin{aligned}
& \Omega_{1}=\left\{x \in E: x(t) \geq q_{2}, \forall t \in J\right\} \\
& \Omega_{2}=\left\{y \in E: y(t) \leq q_{1}, \forall t \in J\right\} .
\end{aligned}
$$

For any $(x, y) \in \Omega_{1} \times \Omega_{2}$ and for all $t \in J$, we have

$$
\|x-y\|_{\infty} \geq|x(t)-y(t)|=x(t)-y(t) \geq\left|q_{1}-q_{2}\right|,
$$

so $\operatorname{dist}\left(\Omega_{1}, \Omega_{2}\right)=\left|q_{1}-q_{2}\right|$. We consider the following two nonlinear functional integral equations 


$$
\begin{aligned}
& x(t)=K(t, x(t))+(T x)(t) \cdot\left(q_{2}+\int_{0}^{t} g(s, y(s)) d s\right) \\
& y(t)=K(t, y(t))+(T y)(t) \cdot\left(q_{1}+\int_{0}^{t} f(s, x(s)) d s\right),
\end{aligned}
$$

where $(x, y) \in \Omega_{1} \times \Omega_{2}$ and $t \in[0,1]$.

The integral equations (FIS1)-(FIS2) may be written, respectively, as:

$$
\begin{aligned}
& x(t)=A x(t) \cdot B y(t)+C x(t) \\
& y(t)=A y(t) \cdot B x(t)+C y(t),
\end{aligned}
$$

where $(x, y) \in \Omega_{1} \times \Omega_{2}$ and $t \in J$. To simplify the notations, we put

$$
\begin{aligned}
& A x(t)=T x(t), \\
& B x(t)=\left(\begin{array}{l}
q_{1}+\int_{0}^{t} f(s, x(s)) d s i f x \in \Omega_{1} \\
q_{2}+\int_{0}^{t} g(s, x(s)) d s i f x \in \Omega_{2},
\end{array}\right. \\
& C x(t)=K(t, x(t)) \text {. }
\end{aligned}
$$

The goal of this section is to apply our main result to investigate the existence of an optimum solution $(x, y)$ of the (FIS1)-(FIS2) problem in the sense that the pair $(x, y)$ satisfies:

$$
\begin{aligned}
\left\|\frac{x-A(x) \cdot B(x)-C(x)}{A(x)}\right\| & =\left\|\frac{y-A(y) \cdot B(y)-C(y)}{A(y)}\right\| \\
& =\operatorname{dist}\left(\Omega_{1}, \Omega_{2}\right) .
\end{aligned}
$$

Note that, if $x$ is a solution of (FIS1) and $y$ is a solution of (FIS2), then the pair $(x, y)$ need not form an optimum solution see [21], pp 27-31 for more details.

We consider the following assumptions:

(i)

(a) The function $K(\cdot, x(\cdot)): J \times \mathbb{R} \longrightarrow \mathbb{R}$ is continuous, for all $x \in E$

(b) There is a continuous function $\delta: J \longrightarrow[0,+\infty)$ with bound $\Delta=\sup _{t \in J}|\delta(t)|$ such that $\mid K(t, x$ $(t))-K(t, y(t))|\leq \delta(t)| x(t)-y(t) \mid$, for all $x, y$ $\in E$ and $t \in[0,1]$ (ii)

(a) The functions $f, g: J \times \mathbb{R} \longrightarrow \mathbb{R}$ are measurables and

$$
N=\max \left\{\sup _{x \in E}\|f(\cdot, x(\cdot))\|_{\infty}, \sup _{x \in E}\|g(\cdot, x(\cdot))\|_{\infty}\right\}<\infty
$$

(b) Let $(x, y) \in \Omega_{1} \times \Omega_{2}$. For all $s \in J$,

$$
|f(s, x(s))-g(s, y(s))| \leq \alpha\left(|x(s)-y(s)|-\left|q_{1}-q_{2}\right|\right)
$$

(c) For all $s \in J, f(s, y(s)) \leq 0 \leq g(s, x(s))$

(iii)

(a) $T: E \longrightarrow E$ is $\mathscr{D}$-Lipschitz with the $\mathscr{D}$-function $\Phi_{T}$, such that $\Phi_{T}$ is differentiable on $\mathbb{R}_{+}$and $\Phi_{A}{ }^{\prime} \mid<1-\Delta / Q+N, N \neq 0$, where $Q=\max \left\{\mid q_{1}\right.$ $\left.|,| q_{2} \mid\right\}$

(b) $T$ is regular on $\mathscr{C}(J, \mathbb{R})$, and $\|T\|=\sup _{x \in E}$ $\|T x\|_{\infty}<1$

(iv) The family $\left\{B(x): x \in \Omega_{1} \cup \Omega_{2}\right\}$ is equicontinuous and closed

Theorem 14. Assume the hypotheses $\left(\mathscr{H}_{1}\right)-\left(\mathscr{H}_{4}\right)$ hold. $\Phi_{T}$ $(r) \leq(1-\|T\|-\Delta / Q+N) r$ for all $r>0$ and $T(x)>0$ for all $x$ $\in$ E. Moreover,

$$
\left(\begin{array}{l}
K(\cdot, x(\cdot)) q_{2} \text { if } x \in \Omega_{1} \\
K(\cdot, x(\cdot)) q_{1} \text { if } x \in \Omega_{2}
\end{array}\right.
$$

Then, there exists an optimum solution $(x, y) \in \Omega_{1} \times \Omega_{2}$ for (FIS1)-(FIS2) problem.

Proof. (1)

(i) By $\left(\mathscr{H}_{1}\right)$, we have $|K(\cdot, x(\cdot))-K(\cdot, y(\cdot))|_{\infty} \leq \Delta$ $\|x-y\|_{\infty}$ for all $x, y \in E$, so $\|C x-C y\|_{\infty} \leq \Phi_{C}($ $\left.\|x-y\|_{\infty}\right)$ for all $x, y \in E$, where $\Phi_{C}(r)=\Delta r$, for all $r$ $\geq 0$

(ii) -Let $x \in \Omega_{1}$, We have, for all $t \in J$

$$
|(B x)(t)|=\left|q_{1}+\int_{0}^{t} f(s, x(s)) d s\right| \leq Q+N .
$$

Similarly, for all $y \in \Omega_{2}$ and $t \in J$ we get $|(B y)(t)| \leq Q+N$. Hence, $M=\left\|B\left(\Omega_{1} \cup \Omega_{2}\right)\right\| \leq Q+N<\infty$. 
(iii) -Furthermore, by hypothesis we have $\Phi_{A}(r) \leq(1-\|$ $A \|-\Delta / Q+N) r$, for all $r>0$. So

$$
\begin{aligned}
M \Phi_{A}(r)+\Phi_{C}(r) & =M \Phi_{A}(r)+\Delta r \leq(Q+N) \Phi_{A}(r)+\Delta r \\
& \leq(1-\|A\|) r, \text { for all } r>0 .
\end{aligned}
$$

(iv) We show that $I-M \Phi_{A}-\Phi_{C}$ is nondecreasing. Let $r, r^{\prime} \in \mathbb{R}_{+}$such that $r<r^{\prime}$.

Since, $\phi_{A}$ is nondecreasing and differentiable on $\mathbb{R}_{+}$, with $\left|\Phi_{A}{ }^{\prime}\right|<1-\Delta / Q+N$, so

$$
0 \leq \frac{\Phi_{A}\left(r^{\prime}\right)-\Phi_{A}(r)}{r^{\prime}-r}<\frac{1-\Delta}{Q+N} \leq \frac{1-\Delta}{M},
$$

thus,

$$
\left(I-M \Phi_{A}-\Phi_{C}\right)(r)<\left(I-M \Phi_{A}-\Phi_{C}\right)\left(r^{\prime}\right) .
$$

That is, $I-M \Phi_{A}-\Phi_{C}$ is nondecreasing.

(2)

(i) Let $t, t^{\prime} \in J$ such that $t<t^{\prime}$,

$$
\begin{aligned}
& \left|B(x)(t)-B(x)\left(t^{\prime}\right)\right| \\
& \quad=\left|\int_{t}^{t} f(s, x(s)) s\right| \leq \int_{t}^{t}|f(s, x(s))| d s \leq N\left|t-t^{\prime}\right|
\end{aligned}
$$

Thus, $B(x)$ is Lipschitzian, so $B(x) \in E$. Let $x \in \Omega_{1}$ and $t$ $\in J$. By $\left(\mathscr{H}_{2}\right)$, (iii), We have

$$
B x(t)=q_{1}+\int_{0}^{t} f(s, x(s)) d s \leq q_{1} .
$$

Hence, $B(x) \in \Omega_{2}$. Similarly, we get $B\left(\Omega_{2}\right) \subset \Omega_{1}$.

(ii) -Let $(x, y) \in \Omega_{2} \times \Omega_{1}$ and $t \in J$

$$
|B x(t)-B y(t)|=\left|\left(q_{1}-q_{2}\right)+\int_{0}^{t}(f(s, x(s))-g(s, y(s))) d s\right| .
$$

By $\left(\mathscr{H}_{2}\right)$, (iii), we have $f(s, x(s))-g(s, y(s)) \leq 0$, for all $s \in J$, so

$$
q_{1}-q_{2}+\int_{J_{2} \cap[0, t]}(f(s, x(s))-g(s, y(s))) d s \leq 0 .
$$

Then,

$$
\begin{aligned}
|B x(t)-B y(t)| & =q_{2}-q_{1}+\int_{0}^{t}(g(s, x(s))-f(s, y(s))) d s \\
& \leq\left|q_{1}-q_{2}\right|+\int_{0}^{t} \alpha\left(|x(s)-y(s)|-\left|q_{1}-q_{2}\right|\right) d s \\
& \leq\left|q_{1}-q_{2}\right|+\int_{0}^{t} \alpha\left(\|x-y\|_{\infty}-\left|q_{1}-q_{2}\right|\right) d s \\
& \leq(1-\alpha)\left|q_{1}-q_{2}\right|+\alpha\|x-y\|_{\infty} .
\end{aligned}
$$

Thus, $\quad\|B x-B y\|_{\infty} \leq \alpha\|x-y\|_{\infty}+(1-\alpha) \operatorname{dist}\left(\Omega_{1}, \Omega_{2}\right)$ which shows that $B$ is cyclic contraction.

(3) Let $y \in \Omega_{2}$ and $x \in E$ such that $x=A(x) \cdot B(y)+C(x)$. We show that $x \in \Omega_{1}$. We have, for all $t \in J, B(y)(t) \geq 0$, $A(x)(t) \geq 0$ and $C(x)(t) \geq q_{2}$, so

$$
x(t)=(A x)(t) \cdot(B y)(t)+(C x)(t) \geq C(x)(t) \geq q_{2} .
$$

Let $x \in \Omega_{1}$ and $y \in E$ such that $y=A(y) \cdot B(x)+C(y)$. We show that $y \in \Omega_{2}$. We have, for all $t \in J, B(y)(t) \leq 0$, $A(x)(t) \geq 0$ and $C(x)(t) \leq q_{1}$, so

$$
y(t)=(A y)(t) \cdot(B x)(t)+(C y)(t) \leq C(x)(t) \leq q_{1} .
$$

(4) As $\left\|B\left(\Omega_{1} \cup \Omega_{2}\right)\right\|<\infty$, so the family $\left\{B(x): x \in \Omega_{1}\right.$ $\left.\cup \Omega_{2}\right\}$ is uniformly bounded; by $\left(\mathscr{H}_{4}\right)$, this family is equicontinuous. Therefore, by Arzela-Ascoli's theorem, $\{B(x): x$ $\left.\in \Omega_{1} \cup \Omega_{2}\right\}$ lies in a compact subset of $\Omega_{1} \cup \Omega_{2}$. Let $\left(x_{n}\right)_{n \geq 0}$ be a sequence of $\Omega_{1} \cup \Omega_{2}$ such that $(I-C / A)^{-1} B\left(x_{n}\right)=$ $x_{n+1}$, i.e., $B\left(x_{n}\right)=(I-C / A)\left(x_{n+1}\right)$. We have $\left(B\left(x_{2 n-1}\right)\right)_{n \geq 1}$ has a convergent subsequence $\left(B\left(x_{2 \sigma(n)-1}\right)\right)_{n \geq 1}$. Let $u=$ $\lim _{n \rightarrow+\infty} B\left(x_{2 \sigma(n)-1}\right)$. As $B\left(\Omega_{1} \cup \Omega_{2}\right)$ is closed, there exists $z \in \Omega_{1} \cup \Omega_{2}$ such that $B(z)=u$. We obtain, for each $n \in \mathbb{N}^{*}$

$$
\begin{aligned}
& \left\|T\left(x_{2 \sigma(n)-1}\right)-T(z)\right\|_{\infty} \\
& \leq\left\|A\left(T\left(x_{2 \sigma(n)-1}\right)\right) \cdot B\left(x_{2 \sigma(n)-1}\right)-A(T(z)) \cdot B(z)\right\|_{\infty} \\
& \quad+\| C\left(T\left(x_{2 \sigma(n)-1}\right)\right)-C\left(T(z) \|_{\infty}\right. \\
& \leq\left\|A\left(T\left(x_{2 \sigma(n)-1}\right)\right)-A(T(z))\right\|_{\infty}\|B\| \\
& \quad+\left\|B\left(x_{2 \sigma(n)-1}\right)-B(z)\right\|_{\infty}\|A\| \\
& \quad+\left\|C\left(T\left(x_{2 \sigma(n)-1}\right)\right)-C(T(z))\right\|_{\infty} \\
& \leq\|A\|\left\|B\left(x_{2 \sigma(n)-1}\right)-B(z)\right\|_{\infty} \\
& \quad+M \Phi_{A}\left(\left\|T\left(x_{2 \sigma(n)-1}\right)-T(z)\right\|_{\infty}\right) \\
& \quad+\Phi_{C}\left(\left\|T\left(x_{2 \sigma(n)-1}\right)-T(z)\right\|_{\infty}\right) .
\end{aligned}
$$


Thus,

$$
\begin{aligned}
& \left\|T\left(x_{2 \sigma(n)-1}\right)-T(z)\right\|_{\infty} \\
& \quad \leq\left(I-M \Phi_{A}-\Phi_{C}\right)^{-1}\left(\|A\|\left\|B\left(x_{2 \sigma(n)-1}\right)-B(z)\right\|_{\infty}\right) \\
& \quad \leq\left\|B\left(x_{2 \sigma(n)-1}\right)-B(z)\right\|_{\infty} .
\end{aligned}
$$

Hence,

$$
\left\|x_{2 \sigma(n)}-T(z)\right\|_{\infty} \leq\left\|B\left(x_{2 \sigma(n)-1}\right)-B(z)\right\|_{\infty} .
$$

Which prove that the sequence $\left(x_{2 \sigma(n)}\right)_{n \geq 0}$ is convergent. that

Thus, by Theorem 1 , there exists $(x, y) \in \Omega_{1} \times \Omega_{2}$ such

$$
\begin{aligned}
& \left\|\frac{x-A(x) \cdot B(x)-C(x)}{A(x)}\right\| \\
& \quad=\operatorname{dist}\left(\Omega_{1}, \Omega_{2}\right)=\left\|\frac{y-A(y) \cdot B(y)-C(y)}{A(y)}\right\| .
\end{aligned}
$$

\section{Data Availability}

No data were used to support this study.

\section{Conflicts of Interest}

The authors declare that there are no conflicts of interest regarding the publication of this paper.

\section{Acknowledgments}

The authors would like to thank the referee for his thorough reviews and valuable remarks, especially for the suggestions to complete references by adding papers $[10,11,13,18]$.

\section{References}

[1] J. Banaś and M. Lecko, "Fixed points of the product of operators in banach algebra," Panamerican Mathematical Journal, vol. 12, no. 2, pp. 101-109, 2002.

[2] J. Banaś and L. Olszowy, "On a class of measures of noncompactness in banach algebras and their application to nonlinear integral equations," Zeitschrift für Analysis und ihre Anwendungen, vol. 28, no. 4, pp. 475-498, 2009.

[3] J. Banaś and K. Sadarangani, "Solutions of some functionalintegral equations in banach algebra," Mathematical and Computer Modelling, vol. 38, no. 3-4, pp. 245-250, 2003.

[4] B. C. Dhage, "A fixed point theorem in banach algebras involving three operators with applications," Kyungpook Mathematical Journal, vol. 44, no. 1, pp. 145-155, 2004.

[5] B. C. Dhage, "On a fixed point theorem in banach algebras with applications," Applied Mathematics Letters, vol. 18, no. 3, pp. 273-280, 2005.

[6] A. Ben Amar, S. Chouayekh, and A. Jeribi, "New fixed point theorems in banach algebras under weak topology features and applications to nonlinear integral equations," Journal of Functional Analysis, vol. 259, no. 9, pp. 2215-2237, 2010.

[7] K. Fan, "Extensions of two fixed point theorems of F. E. browder," Mathematische Zeitschrift, vol. 112, no. 3, pp. 234-240, 1969.

[8] A. A. Eldred and P. Veeramani, "Existence and convergence of best proximity points," Journal of Mathematical Analysis and Applications, vol. 323, no. 2, pp. 1001-1006, 2006.

[9] M. A. Al-Thagafi and N. Shahzad, "Best proximity sets and equilibrium pairs for a finite family of multimaps," Fixed Point Theory and Applications, vol. 2008, no. 1, Article ID 457069, 2008.

[10] M. Gabeleh and J. Markin, "Noncyclic meir-keeler contractions and best proximity pair theorems," Demonstratio Mathematica, vol. 51, no. 1, pp. 171-181, 2018.

[11] M. Gabeleh and H.-P. A. Künzi, "Equivalence of the existence of best proximity points and best proximity pairs for cyclic and noncyclic nonexpansive mappings," Demonstratio Mathematica, vol. 53, no. 1, pp. 38-43, 2020.

[12] K. Chaira and S. Lazaiz, "Best proximity point theorems for cyclic Relatively $\rho$-Nonexpansive mappings in modular spaces," Abstract and Applied Analysis, vol. 2018, 8 pages, 2018.

[13] M. M. Gabeleh, P. Moshokoa, and C. Vetro, "Cyclic (noncyclic) phi-condensing operator and its application to a system of differential equations," Nonlinear Analysis: Modelling and Control, vol. 24, no. 6, pp. 985-1000, 2019.

[14] N. Hussain, M. Hezarjaribi, M. A. Kutbi, and P. Salimi, "Best proximity results for suzuki and convex type contractions," Fixed Point Theory and Applications, vol. 2016, no. 1, p. 14, 2016.

[15] N. Hussain, A. Latif, and P. Salimi, "Best proximity point results for modified suzuki $\alpha-\psi$-proximal contractions," Fixed Point Theory and Applications, vol. 2014, no. 1, 2014.

[16] A. Latif, M. Hezarjaribi, P. Salimi, and N. Hussain, "Best proximity point theorems for $\alpha$ - $\psi$-proximal contractions in intuitionistic fuzzy metric spaces," Journal of Inequalities and Applications, vol. 2014, no. 1, 2014.

[17] V. Sankar Raj and P. Veeramani, "Best proximity pair theorems for relatively nonexpansive mappings," Applied General Topology, vol. 10, no. 1, pp. 21-28, 2009.

[18] F. Tchier, C. Vetro, and F. Vetro, "Best approximation and variational inequality problems involving a simulation function," Fixed Point Theory and Applications, vol. 2016, no. 1, Article ID 26, 2016.

[19] A. A. Eldred, W. A. Kirk, and P. Veeramani, "Proximal normal structure and relatively nonexpansive mappings," Studia Mathematica, vol. 171, no. 3, pp. 283-293, 2005.

[20] D. W. Boyd and J. S. W. Wong, "On nonlinear contractions," Proceedings of the American Mathematical Society, vol. 20, no. 2, pp. 458-464, 1969.

[21] Q. H. Ansari, Nonlinear Analysis: Approximation Theory, Optimization and Applications, Springer, 2014. 